Research Article

\title{
Interface Slip of the Steel Beam Strengthened with the CFRP Sheet under Temperature and Creep
}

\author{
Wenyu Hou, Yanfeng Li (iD, and Yongping Guan \\ School of Transportation Engineering, Shenyang Jianzhu University, Shenyang 110168, China \\ Correspondence should be addressed to Yanfeng Li; lyfneu@126.com
}

Received 31 May 2021; Accepted 14 June 2021; Published 22 June 2021

Academic Editor: Luigi Di Sarno

Copyright (C) 2021 Wenyu Hou et al. This is an open access article distributed under the Creative Commons Attribution License, which permits unrestricted use, distribution, and reproduction in any medium, provided the original work is properly cited.

\begin{abstract}
This paper studied the interface slip between the CFRP sheet and the steel beam under the action of temperature and creep. The formulas of the interface slip were established by using the elastic method and energy variational method, respectively, and the accuracy of the formulas was verified by finite element analysis Abaqus. The results showed that when the creep was not taken into account, the interface slip was the maximum at the end of the beam and the minimum at the middle of the span. When the temperature increased to $5^{\circ} \mathrm{C}$, the slip increased about $0.005 \mathrm{~mm}$. And when the thickness of the CFRP sheet increased to $0.05 \mathrm{~mm}$, the slip increased about $0.003 \mathrm{~mm}$. When the creep was taken into account, the interface slip increased with the increase of load and decreased with the increase of stiffness. When the stiffness reached about $10 \mathrm{ka}$, the variation of the stiffness on the slip was basically unchanged.
\end{abstract}

\section{Introduction}

Relative slip will occur between CFRP material and steel structure when the steel structure is reinforced with CFRP material. This slip may lead to peeling failure of the interface. Previous studies [1] showed that the interface peeling failure was an important reason why the CFRP materials cannot achieve the effect of strengthening and repairing steel structures. The interface slip between the interface of the steel beam and the CFRP sheet will occur under the action of temperature and creep when the steel beam is strengthened with the CFRP sheet. The interface slip will affect the interfacial interaction, reduce the bearing capacity and stiffness, and increase the deflection of the beam. Some experts have studied the interfacial mechanical properties of steel structures strengthened with CFRP materials.

Nguyen et al. [2] showed that the strength and stiffness of the adhesive between the interface of the CFRP plate and steel would degenerate lower than $10 \%$ in the environment with relative humidity of $90 \%$ and a temperature of $20-50^{\circ} \mathrm{C}$ when cycled for 1000 hours.

$\mathrm{Yu}$ et al. [3] conducted an experimental study on the interface properties of CFRP plates and steel plates. The type of adhesive, the thickness of the adhesive layer, and the stiffness of CFRP plates were taken as parameters. The results showed that the bonding strength was closely related to the interfacial fracture energy. The bond strength was positively correlated with the stiffness of the CFRP plate. When the thickness of the adhesive layer was between 1 and $2 \mathrm{~mm}$, the bonding strength would increase with the increase of the thickness. The effect of CFRP bonding length on the interface bonding performance was mainly reflected in that it was within the range of effective bonding length, and the effect beyond this range was very small.

Jiang [4] studied the interfacial properties of flexural steel beams strengthened by the CFRP plate and analyzed the influence of various factors on the reinforced members. When the prestressed CFRP plate was used to strengthen the beams, the bearing capacity could be improved by increasing the thickness of the CFRP, but the stiffness almost did not increase.

Fernando et al. [5] found that when the adhesive adopts elastic adhesive, the constitutive models of the interface between CFRP and steel and the interface between FRP and concrete were very similar and presented a triangle shape. On the basis of the simplified FRP- 
concrete interface constitutive model proposed by Lu, Fernando et al. gave a constitutive model for the interface between CFRP and steel.

Al-Shawaf and Zhao [6] conducted an experimental study on the interfacial properties of CFRP-steel doubleshear specimens under wet dressing and low temperature. It was found that the interface failure of the adhesive layer mainly occurred in the low-temperature environment. The effective bond length, axial strain distribution, and interfacial shear stress distribution of the CFRP-steel interface have little influence on the maintenance environment, and the increase of temperature could effectively improve the bond strength.

Zhao et al. [7] improved the bonding method. By increasing the bond length of the stressed side $(L 1>L 2)$, the load was guaranteed to occur on the side with short bond length. This improved method could significantly reduce the number of measuring points, but because the bonding was in two interfaces, the samples should be cured separately, increasing the test period, and the thickness of the bonding layer on both sides should be kept consistent as far as possible.

Xue [8] conducted an experimental study on the interface properties of CFRP-steel with the parameters length, width, number of layers of CFRP, and curing time. The results showed that the improvement of the ultimate bearing capacity of specimens was significantly affected by the bonding width. In order to improve the ultimate bearing capacity of the interface, the effective bond length could be effectively increased by increasing the number of CFRP layers. The peak strain of CFRP and the ultimate bearing capacity of the specimen would remain basically unchanged since the CFRP bonding length reached the effective bonding length. When the curing time of the specimen becomes longer, the effective transfer length of the interface would decrease, and the shear stress of the adhesive layer would increase.

Teng et al. [9] and Yuan [10] used the cohesion element in Abaqus to simulate the interface behavior of CFRP and steel based on fracture mechanics. There were two advantages of using the cohesion element. One was that the cohesion element sets the geometric thickness in the model. The difference between the cohesion element and the solid element was that there was no need to divide the mesh along the thickness direction, and the calculation accuracy was still high. Secondly, the stress distribution of the interface along the thickness direction can be obtained by setting the geometric thickness. The disadvantage was that this element was only suitable for the bilinear bond-slip model, but not for other bond-slip models.

Wang et al. [11] used 3D digital imaging technology (3DDIC) to measure the shear stress and slip of the nonlinear adhesive interface and obtained the bond-slip relationship, which further proved the rationality of using the trilinear bond-slip model of the nonlinear adhesive interface. Since there were only 6 experimental data, the expression of the key parameters was not given. Therefore, more data were needed to obtain a bond-slip model for the nonlinear adhesive interface.
Heshmati et al. [12] used the double-sided shear test to study the mechanical behavior of the CFRP-steel interface and GFRP-steel interface in the distillation water/deicing salt solution. The results showed that the bond stiffness and strength of the interface between the two materials and steel were obviously reduced. The distribution of water on the FRP-steel interface was simulated by the finite element method, and the influence mechanism of water molecules on the interfacial bond property of CFRP-steel was analyzed.

$\mathrm{Li}$ et al. [13] carried out tensile shear tests on 17 CFRP plate and steel single-lap specimens. Studying the mechanical behavior and interfacial failure mode of CFRP-steel between different epoxy binders and CFRP materials, the effects of the type of binder and CFRP materials on the interfacial bond-slip constitutive and interfacial shear properties were analyzed. The results showed that the interface failure modes and shear capacity were different with different binder or CFRP materials.

Pang [14] studied interlaminar detachment failure and mixed failure modes of CFRP plates under the extreme service environment. The results showed that the interfacial bond property was only related to the binder properties when the mixing failure occurred. However, when the interlaminar detachment failure of CFRP plates occurred, the interfacial bonding property was related to both the binder and the properties of CFRP plates.

Wei et al. [15] conducted 12 double-shear joint tests of the CFRP plate and steel and analyzed the failure mode, ultimate load, CFRP plate strain, interfacial shear stress, and bond-slip relationship. The test results showed that freezethaw cycles had no effect on the interface failure mode. The freeze-thaw environment had no effect on the strain distribution of the CFRP plate, interfacial shear stress distribution, and shape of the curve of the bond-slip relationship, but the maximum strain of the CFRP plate, maximum interfacial shear stress, and maximum slip would decrease due to the freeze-thaw cycle.

$\mathrm{Xu}$ et al. [16] carried out long-term loading tests of CFRP-steel double-shear tests with 4 interfacial shear stress levels and 6 kinds of holding times in order to study the influence of loading history on the interfacial bond properties of steel structures reinforced by CFRP and carried out static tensile failure on the specimens that reached the specified loading time. The results showed that the creep deformation of adhesives could cause the redistribution of interfacial stress. With the increase of time, the CFRP strain increased, and the peak value of interfacial shear stress decreased. When the level of interfacial shear stress is high, the influence of interfacial creep damage on the bond-slip curve should be considered. When the bond length of CFRP was larger than the effective bond length, the interface creep damage had no obvious effect on the ultimate bearing capacity.

$\mathrm{Xu}$ et al. [17] carried out long-term loading tests on CFRP-steel double-shear tests under different tensile loads in order to study the creep characteristics of CFRP-steel interface adhesive. The results showed that the CFRP-steel interface adhesive exhibited creep deformation under longterm interfacial shear stress. The CFRP strain decreased 
nonlinearly from the loading end to the fixed end and increased with the increase of loading time, and the rate of increase decreased gradually.

It can be found that the analysis of the interface slips under the influence of temperature and creep is relatively rare based on the research studies. This paper gave the calculation formula of the interface slip between the CFRP sheet and the steel beam under the effect on temperature and creep using the elastic method and energy variational method, and the accuracy of the formula was verified by Abaqus. Then, the influences of design parameters were discussed.

\section{Elastic Method}

Based on the elastic method [18] and the mechanical characteristics of the steel beam strengthened with the CFRP sheet, the following assumptions were made: (1) the reinforced steel beam was an elastomer in normal service condition; (2) the interface adhesive layer only bore shear force; (3) CFRP sheet only bore tensile force; (4) the section conformed to the plane cross-section assumption. The element of the CFRP sheetstrengthened steel beam is shown in Figure 1.

The equilibrium equation can be obtained from the element force:

$$
M_{s}-\left(M_{s}+\mathrm{d} M_{s}\right)+V_{s} \mathrm{~d} x+\tau \mathrm{d} x \cdot y_{s}=0 .
$$

According to the relationship between bending moment and curvature,

$$
\frac{\mathrm{d} \phi}{\mathrm{d} x}=\frac{1}{E_{s} I_{s}} \frac{\mathrm{d} M_{s}}{\mathrm{~d} x} .
$$

Strain on the lower surface of the lower flange of the steel beam is

$$
\begin{aligned}
\varepsilon_{s}(x) & =\phi y_{s}-\frac{N_{s}}{E_{s} A_{s}} \\
& =\phi y_{s}-\frac{N_{0}+P_{T_{s}}}{E_{s} A_{s}} .
\end{aligned}
$$

Strain on the surface of the CFRP sheet is

$$
\varepsilon_{p}(x)=\frac{N_{p}}{E_{p}(T, t) A_{p}},
$$

where $N_{0}, V_{s}$, and $M_{s}$ are the axial force, shear force, and bending moment caused by external loading. $\tau$ is the interfacial shear stress. $P_{T_{s}}$ is the axial force due to temperature changes [19].

So, the following can be available:

$$
\begin{aligned}
\frac{\mathrm{d} s(x)}{\mathrm{d} x} & =\varepsilon_{s}(x)-\varepsilon_{p}(x) \\
& =y_{s} \phi-\left(\frac{N_{s}}{E_{s} A_{s}}+\frac{N_{p}}{E_{p}(T, t) A_{p}}\right), \\
\frac{\mathrm{d}^{2} s(x)}{\mathrm{d} x^{2}} & =y_{s} \frac{\mathrm{d} \phi}{\mathrm{d} x}-\left(\frac{1}{E_{s} A_{s}} \frac{\mathrm{d} N_{s}}{\mathrm{~d} x}+\frac{1}{E_{p}(T, t) A_{p}} \frac{\mathrm{d} N_{p}}{\mathrm{~d} x}\right) .
\end{aligned}
$$

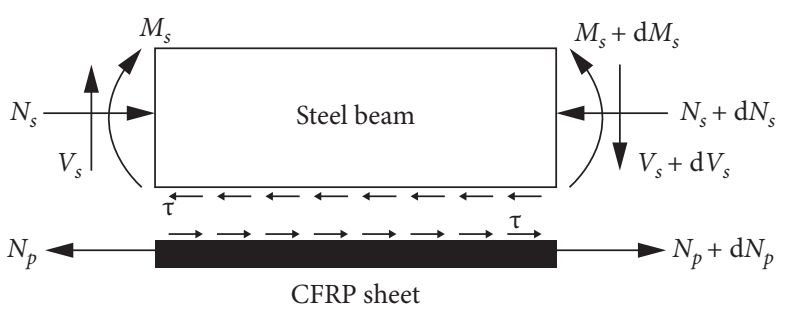

Figure 1: The element force.

Because $N_{s}=N_{p},(\mathrm{~d} N / \mathrm{d} x)=-\tau$, and $\tau=k_{a} s(x)$, the differential equation of the interface slip can be obtained:

$$
\frac{\mathrm{d}^{2} s(x)}{\mathrm{d} x^{2}}-\alpha^{2} s(x)-\beta V_{s}+\gamma \frac{\mathrm{d} N_{0}}{\mathrm{~d} x}+\gamma \frac{\mathrm{d} P_{T_{s}}}{\mathrm{~d} x}=0,
$$

where $\alpha^{2}=k_{a}\left(\left(y_{s}^{2} / E_{s} I_{s}\right)+\left(1 / E_{p}(T, t) A_{p}\right)\right) ; \quad \beta=y_{s} / E_{s} I_{s}$; $\beta_{\tau}=k_{a} y_{s} / E_{s} I_{s} ; \quad \gamma=1 / E_{s} A_{s} ; \quad \gamma_{\tau}=k_{a} / E_{s} A_{s} ; \quad k_{a}=b_{p} G_{a} / t_{a} ;$ $E_{p}(T, t)=\phi(T, t) E_{p} ; P_{T_{s}}=E_{s} \alpha_{s} \int_{h} t(y) b(y) \mathrm{d} y ; E_{s}, E_{p}(T, t)$ are the elastic modulus of the steel beam and CFRP sheet; $A_{s}$, $A_{p}$ are the cross-section area of the steel beam and CFRP sheet; $I_{s}$ is the moment of inertia of the steel beam; $y_{s}$ is the distance between the neutral shaft and the bottom of the steel beam; $\alpha_{S}$ is the expansion coefficient of the steel beam; $t(y)$ is the temperature function of the steel beam; $b(y)$ is the width; $G_{a}$ is the stiffness of the adhesive layer; $t_{a}$ is the thickness of the adhesive layer; $E_{p}$ is the initial modulus of elasticity of the CFRP sheet; $\phi(T, t)$ is the influence coefficient of temperature and creep of the CFRP sheet $[20], \phi(T, t)=[1 / 1+$ $\left.0.1 \sinh \left(\left(T / T_{0}\right)-1\right) t^{0.05}\right]+\left(1 / 1+\left(5 / \beta_{1}\right) t^{0.2}\right)-1 ; \beta_{1}$ is the ratio of creep modulus to elastic modulus, can be taken as 45 ; $T$ is the temperature value; $T_{0}$ is the initial temperature; and $t$ is the hour.

\section{Energy Variational Method}

Based on the energy variational method [21], it was assumed that, under the action of external loading, the displacement of the steel beam was $U_{s}$, the displacement of the CFRP sheet was $U_{p}$, the deformation of the beam was $W$, and the displacement of the adhesive layer was $\Delta_{t}=U_{s}-y_{s} W^{\prime}-U_{p}$.

Strain energy of the steel beam is

$$
\prod_{s}=\frac{1}{2} E_{s} A_{s} \int U_{s}^{\prime 2} \mathrm{~d} x+\frac{1}{2} E_{s} I_{s} \int W^{\prime \prime 2} \mathrm{~d} x .
$$

Strain energy of the CFRP sheet is

$$
\prod_{p}=\frac{1}{2} E_{p}(T, t) A_{p} \int U_{p}^{\prime 2} \mathrm{~d} x+\frac{1}{2} E_{p} I_{p} \int W^{\prime \prime 2} \mathrm{~d} x .
$$

Strain energy of the adhesive layer is

$$
\prod_{t}=\frac{1}{2} \int k_{a}\left(U_{s}-U_{p}-y_{s} W^{\prime}\right)^{2} \mathrm{~d} x .
$$

The work of external loading is 


$$
\prod_{0}=\int M W^{\prime \prime} \mathrm{d} x
$$

$$
\begin{aligned}
\prod= & \frac{1}{2} E_{s} A_{s} \int U_{s}^{\prime 2} \mathrm{~d} x+\frac{1}{2} E_{s} I_{s} \int W^{\prime \prime 2} \mathrm{~d} x+\frac{1}{2} E_{p}(T, t) A_{p} \int U_{p}^{\prime 2} \mathrm{~d} x+\frac{1}{2} E_{p}(T, t) I_{p} \int W^{\prime \prime 2} \mathrm{~d} x \\
& +\frac{1}{2} k_{a}\left(U_{s}-U_{p}-y_{1} W^{\prime}\right)^{2} d x+\int M W^{\prime \prime} \mathrm{d} x .
\end{aligned}
$$

According to the principle of minimum potential energy, the following can be obtained:

$$
\begin{aligned}
\delta \prod= & E_{s} A_{s} \int U_{s}^{\prime} \delta U_{s} \mathrm{~d} x+E_{s} I_{s} \int W \delta W^{\prime \prime} \mathrm{d} x+E_{p}(T, t) A_{p} \int U_{p}^{\prime} \delta U_{p} \mathrm{~d} x+E_{p}(T, t) I_{p} \int W^{\prime \prime} \delta W^{\prime \prime} \mathrm{d} x \\
& +\int k_{a}\left(U_{s}-U_{p}-y_{s} W^{\prime}\right)\left(\delta U_{s}-\delta U_{p}-y_{s} \delta W^{\prime \prime}\right) d x+\int M \delta W^{\prime \prime} \mathrm{d} x=0 .
\end{aligned}
$$

And $\delta U_{s}$ and $\delta U_{p}$ are independent, so

$$
\begin{aligned}
& \int\left[-E_{p}(T, t) A_{p} U_{p}^{\prime \prime}-k_{a}\left(U_{s}-U_{p}-y_{s} W^{\prime}\right)\right] \delta U_{p}=0, \\
& \int\left[-E_{s} A_{s} U_{s}^{\prime \prime}+k_{a}\left(U_{s}-U_{p}-y_{s} W^{\prime}\right)\right] \delta U_{s}=0, \\
& \int\left[E_{s} I_{s} W^{(4)}+E_{p}(T, t) I_{p} W^{(4)}+k_{a} y_{s}\left(U_{s}-U_{p}-y_{s} W^{\prime}\right)-M^{\prime \prime}\right] \delta W=0 .
\end{aligned}
$$

According to internal force equilibrium conditions,

$$
\begin{aligned}
N_{s} & =N_{0}+P_{T_{s}} \\
& =E_{s} A_{s} U_{s}^{\prime}, \\
N_{p} & =E_{p}(T, t) A_{p} U_{p}^{\prime} .
\end{aligned}
$$

The following can be obtained:

$$
\frac{\mathrm{d}^{2} s(x)}{\mathrm{d} x^{2}}-\alpha^{2} s(x)-\beta V(x)+\gamma \frac{\mathrm{d} N_{0}}{\mathrm{~d} x}+\gamma \frac{\mathrm{d} P_{T s}}{\mathrm{~d} x}=0 .
$$

\section{Interface Slip Formula}

Under the action of uniform load, symmetrical load, and concentrated loading (the distance between the position of the load and the midspan was $b$ ), the length of the beam was $L$, and the paste length of the CFRP sheet was $l_{f}$; taking the midspan as the origin of coordinates, down was the $y$-direction, and the right was the $x$-direction; taking half of the beam as the research object and combining boundary conditions, the interface slip formulas under different loads can be obtained.

(1) Under uniform load,

$$
s(x)=\frac{e^{\alpha x}-e^{-\alpha x}}{\alpha^{3}\left(e^{\left(\alpha l_{f} / 2\right)}+e^{-\left(\alpha l_{f} / 2\right)}\right)}\left\{\beta q+\alpha^{2}\left[\frac{P_{T_{s}}}{E_{s} A_{s}}-\frac{q y_{s} L\left(L-l_{f}\right)}{4 E_{s} I_{s}}\right]\right\}-\frac{\beta q}{\alpha^{2}} x .
$$

(2) Under symmetrical loading,

Bending and shear section: 


$$
\begin{aligned}
s_{1}(x)= & \frac{\beta P\left(e^{\alpha l_{0} / 2}+e^{-\left(\alpha l_{0} / 2\right)}\right)}{2 \alpha^{2}\left(e^{\alpha l_{f} / 2}+e^{-\left(\alpha l_{f} / 2\right)}\right)}\left[e^{\alpha\left(\left(l_{f} / 2\right)-x\right)}+e^{-\alpha\left(\left(l_{f} / 2\right)-x\right)}\right] \\
& +\left[\frac{P_{T_{s}}}{E_{s} A_{s}}-\frac{P y_{s}\left(L-l_{f}\right)}{2 E_{s} I_{s}}\right] \frac{e^{\alpha x}-e^{-\alpha x}}{\alpha\left(e^{\alpha l_{f} / 2}+e^{-\left(\alpha l_{f} / 2\right)}\right)}-\frac{\beta P}{\alpha^{2}} .
\end{aligned}
$$

Bending section:

$$
s_{2}(x)=\frac{e^{\alpha x}-e^{-\alpha x}}{e^{\alpha l_{f} / 2}+e^{-\left(\alpha l_{f} / 2\right)}}\left\{\alpha\left[\frac{P_{T s}}{E_{s} A_{s}}-\frac{P y_{s}\left(L-l_{f}\right)}{2 E_{s} I_{s}}\right]-\frac{\beta P}{2 \alpha^{2}}\left[e^{\alpha\left(\left(l_{f} / 2\right)-\left(l_{0} / 2\right)\right)}-e^{-\alpha\left(\left(l_{f} / 2\right)-\left(l_{0} / 2\right)\right)}\right]\right\} .
$$

(3) Under concentrated loading,

Left side of the loading point:

$$
\begin{aligned}
s_{1}(x)= & \frac{\beta((P / 2)-(P b / L))\left[e^{\alpha\left(\left(l_{f} / 2\right)-x\right)}+e^{-\alpha\left(\left(l_{f} / 2\right)-x\right)}\right]+\alpha\left[\left(P_{T_{s}} / E_{s} A_{s}\right)-\left(\left(y_{s}\left(L-l_{f}\right) / 2 E_{s} I_{s}\right)((P / 2)-(P b / L))\right)\right]\left(e^{\alpha x}-e^{-\alpha x}\right)}{\alpha^{2}\left(e^{\alpha l_{f} / 2}+e^{-\alpha l_{f} / 2}\right)} \\
& -\frac{\beta}{\alpha^{2}}\left(\frac{P}{2}-\frac{P b}{L}\right) .
\end{aligned}
$$

Right side of the loading point:

$$
\begin{aligned}
s_{2}(x)= & \frac{\beta((P / 2)+(P b / L))\left[e^{\alpha\left(\left(l_{f} / 2\right)-x\right)}+e^{-\alpha\left(\left(l_{f} / 2\right)-x\right)}\right]+\alpha\left[\left(P_{T_{s}} / E_{s} A_{s}\right)-\left(y_{s}\left(L-l_{f}\right) / 2 E_{s} I_{s}\right)((P / 2)+(P b / L))\right]\left(e^{\alpha x}-e^{-\alpha x}\right)}{\alpha^{2}\left(e^{\left(\alpha l_{f} / 2\right)}+e^{-\left(\alpha l_{f} / 2\right)}\right)} \\
& -\frac{\beta}{\alpha^{2}}\left(\frac{P}{2}+\frac{P b}{L}\right) .
\end{aligned}
$$

\section{Numerical Analysis}

5.1. Constitutive Relation. In order to verify the accuracy of the theoretical calculation, this paper used finite element calculation software Abaqus to check the calculation. The constitutive relation of the steel beam in the Abaqus model adopted the plastic analysis model. Figures 2 and 3 show the constitutive relationship of steel and CFRP sheet, and Poisson's ratio of steel is 0.3 . CFRP sheet was an orthotropic material with no strength in the vertical fiber direction. The stress and strain relation curve of CFRP cloth was taken as ideal elasticity, and the elastic modulus of CFRP cloth was consistent with the value in the previous formula.

5.2. Calculation Results. Figures 4 and 5 show the strain distribution of the bottom flange of the steel beam and CFRP sheet under the load of $10 \mathrm{~N} / \mathrm{mm}$ and the temperature of $20^{\circ} \mathrm{C}$ and $25^{\circ} \mathrm{C}$. Because the interface slip between the steel beam and the CFRP sheet cannot be obtained directly in Abaqus, the difference of strain between the steel beam and the CFRP sheet is used to calculate the interface slip.

Figure 6 shows the comparison between the finite element calculation and the formula calculation results. It showed that the two results were in good agreement and indicated that the formula was correct and can be used to calculate the interface slip between the steel beam and the CFRP sheet.

\section{Parameter Analysis}

6.1. Effect of Adhesive Stiffness. Figure 7 shows the interface slip distribution curve along the beam length of different adhesive stiffness. The curves were nonlinear, and the 


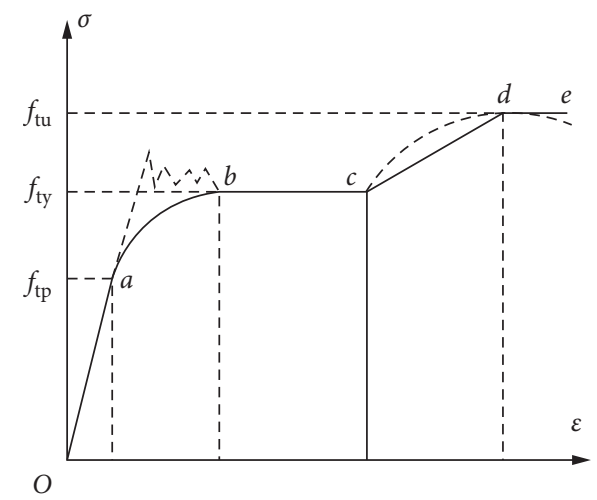

FIGURE 2: Stress-strain curve of steel.

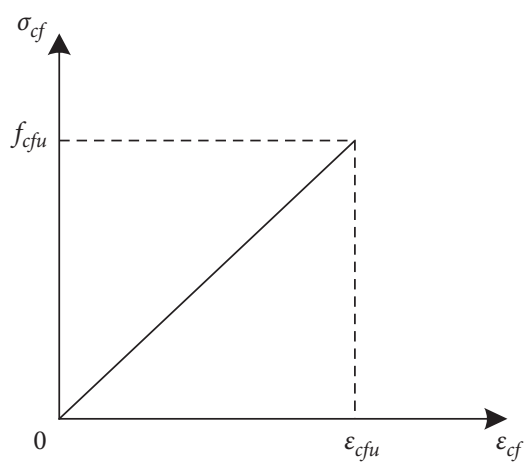

FIGURE 3: Stress-strain curve of the CFRP sheet.
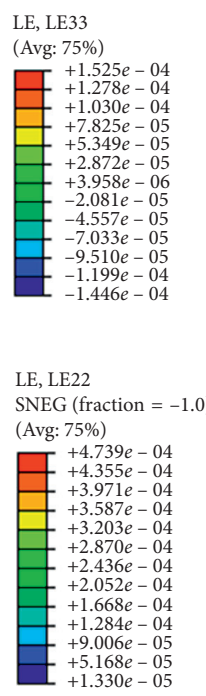

(a)

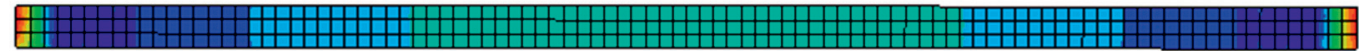

(b)

Figure 4: Strain distribution under the temperature of $20^{\circ} \mathrm{C}$. (a) Bottom flange of the steel beam. (b) CFRP sheet.

interface slip decreased with the increase of the stiffness. The change of the interface slip gradually decreased with the increase of the stiffness. When the stiffness reached $3 \mathrm{ka}$, the change of the interface slip was very small, indicating that the increase of the stiffness had basically no effect on the interface slip after the stiffness exceeded $3 \mathrm{ka}$. 


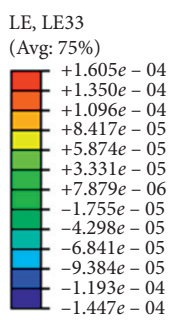

LE, LE22

SNEG (fraction $=-1.0$

(Avg: $75 \%$ )

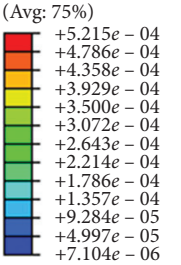

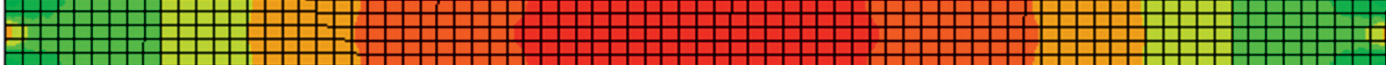

(a)

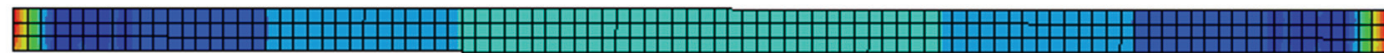

(b)

FIgUre 5: Strain distribution under the temperature of $25^{\circ} \mathrm{C}$. (a) Bottom flange of the steel beam. (b) CFRP sheet.

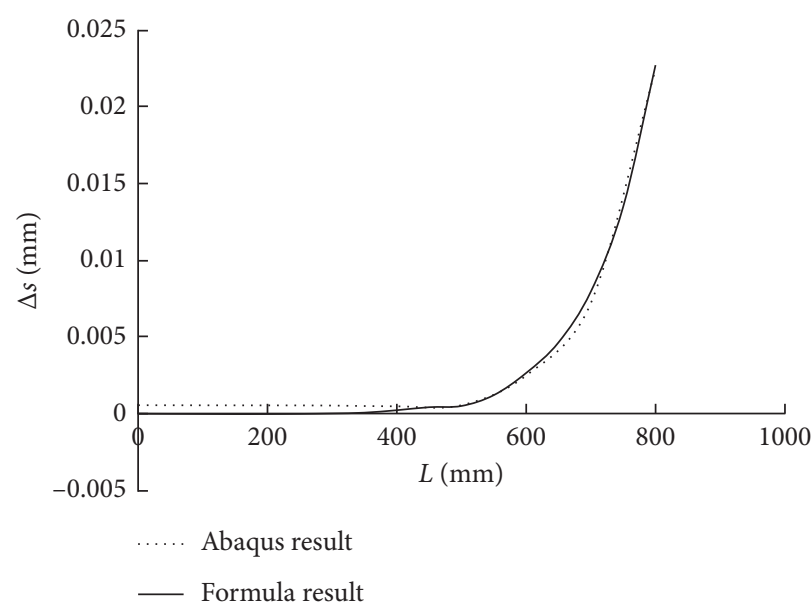

(a)

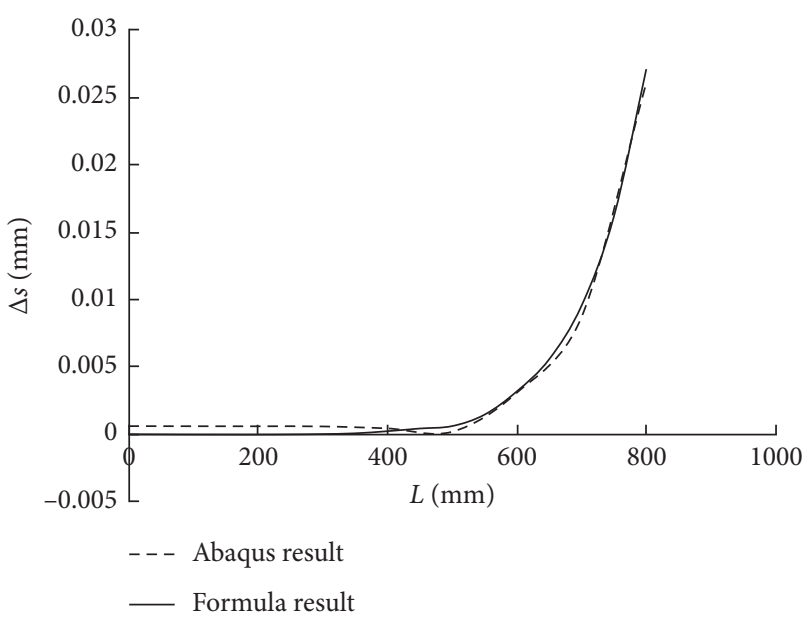

(b)

Figure 6: The comparison between numerical results and calculation results. (a) Temperature of $20^{\circ} \mathrm{C}$. (b) Temperature of $25^{\circ} \mathrm{C}$.

6.2. Effect of Temperature. Figure 8 shows the interface slip distribution curve along the beam length of different temperatures. The curves were nonlinear. The higher the temperature was, the greater the interface slip was. The maximum slip was at the end of the beam. When the temperature increased to $5^{\circ} \mathrm{C}$, the slip increased about $0.005 \mathrm{~mm}$.

6.3. Effect of Thickness of the CFRP Sheet. Figure 9 shows the interface slip distribution curve along the beam length of different thicknesses of the CFRP sheet. The curves were nonlinear. The greater the thickness of the CFRP sheet was, the greater the interface slip was. The maximum slip was at the end of the beam. When the thickness of the CFRP sheet increased to $0.05 \mathrm{~mm}$, the slip increased about $0.003 \mathrm{~mm}$.
6.4. Effect of Loading under CFRP Creep. Figure 10 shows the interface slip distribution curve along with the time of different loads. The curves were nonlinear. At the beginning of loading, the slip was 0 , and the slip increment increased with the increase of time. The slip increased with the increase of load. The larger the load was, the steeper the curve was. When the load increased to $5 \mathrm{~N} / \mathrm{mm}$ or $5 \mathrm{kN}$, the slip increased about $4.4 \times 10^{-7} \mathrm{~mm}, \quad 1.2 \times 10^{-6} \mathrm{~mm}$, and $6.2 \times 10^{-7} \mathrm{~mm}$, respectively.

6.5. Effect of Adhesive Stiffness under Creep. Figure 11 shows the interface slip distribution curve along with the time of different adhesive stiffness. The curves were nonlinear. The slip decreased with the increase of stiffness. The greater the stiffness was, the gentler the curve was. 


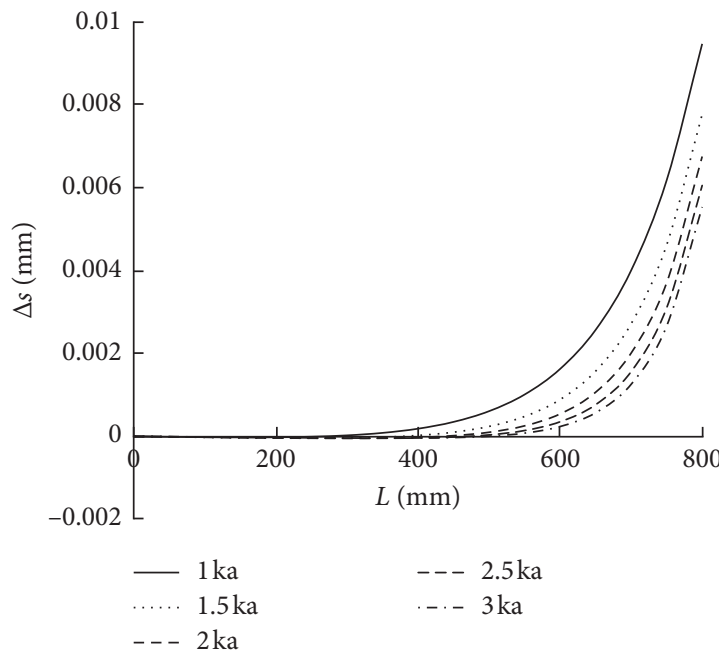

(a)

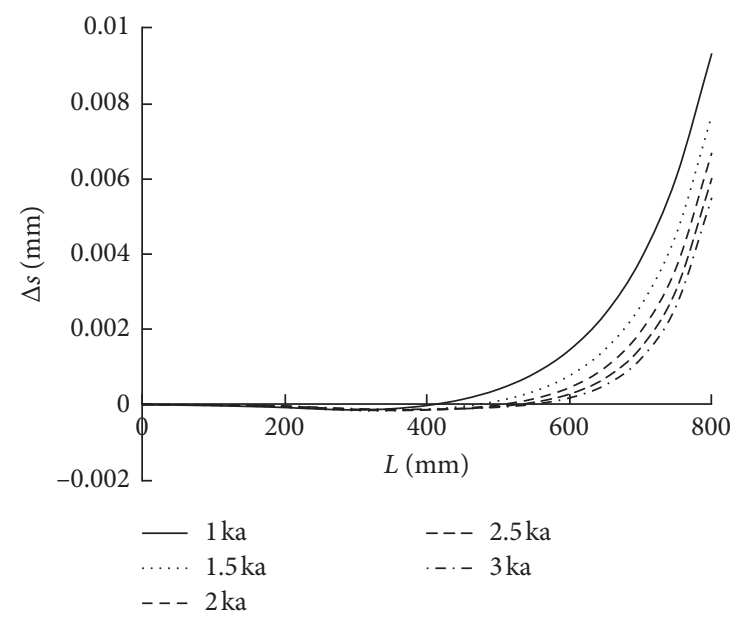

(b)

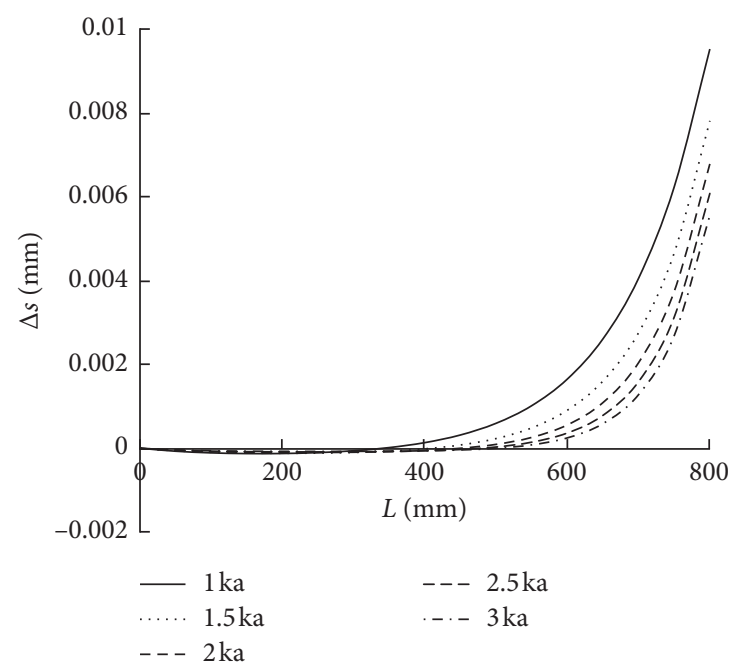

(c)

Figure 7: The effect of adhesive layer stiffness on the interface slip. (a) Uniform load. (b) Symmetrical concentrated load. (c) Midspan concentrated load.

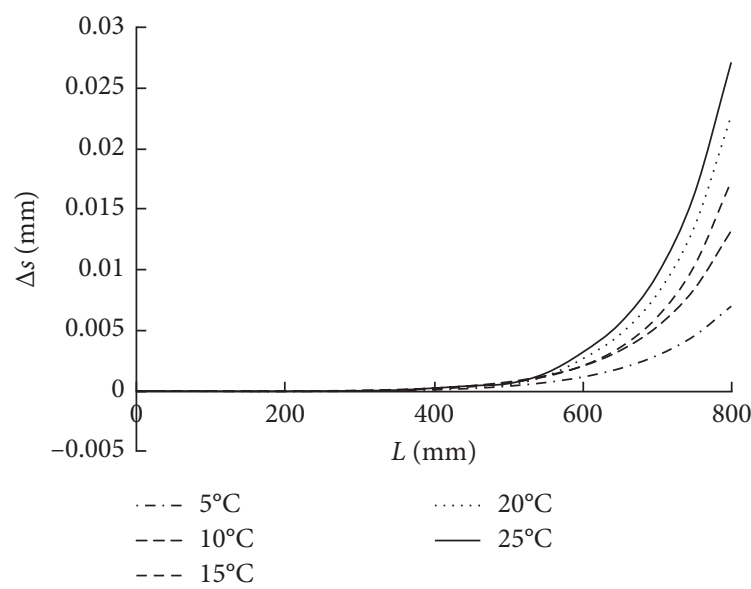

(a)
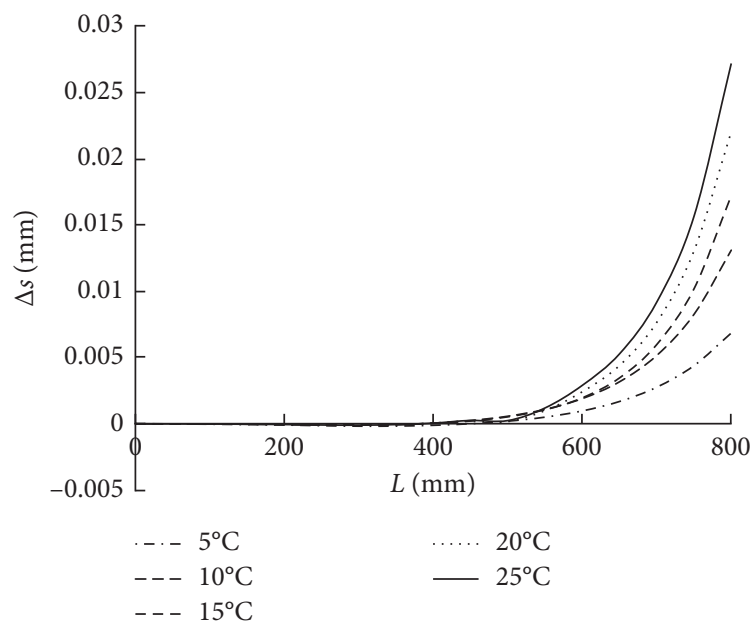

(b)

Figure 8: Continued. 


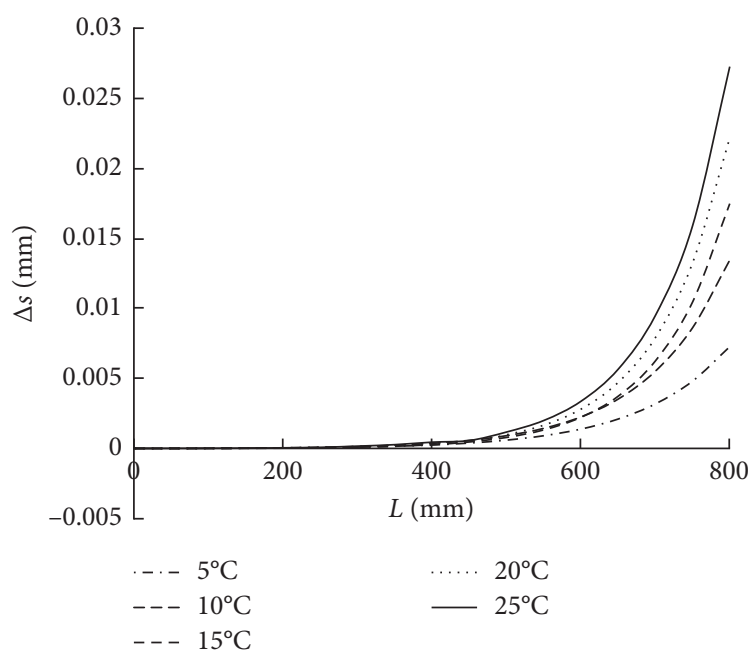

(c)

FIGURE 8: The effect of temperature on the interface slip. (a) Uniform load. (b) Symmetrical concentrated load. (c) Midspan concentrated load.

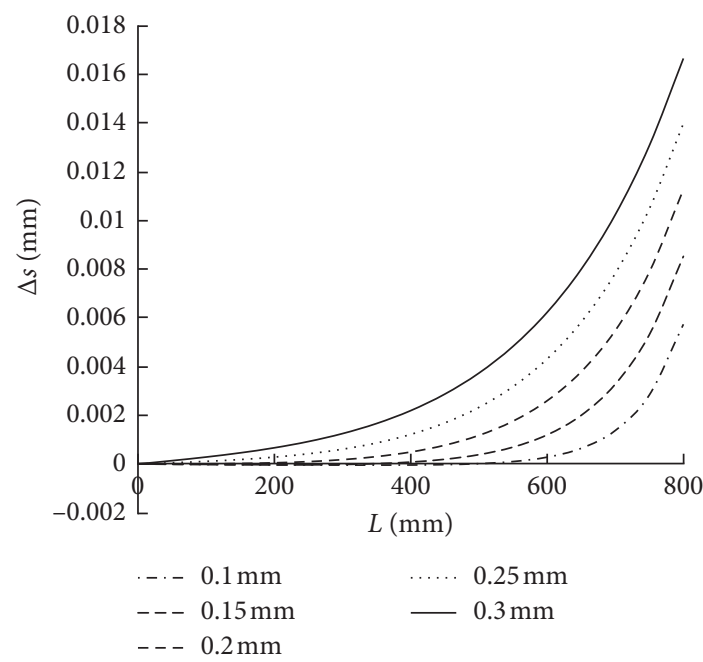

(a)

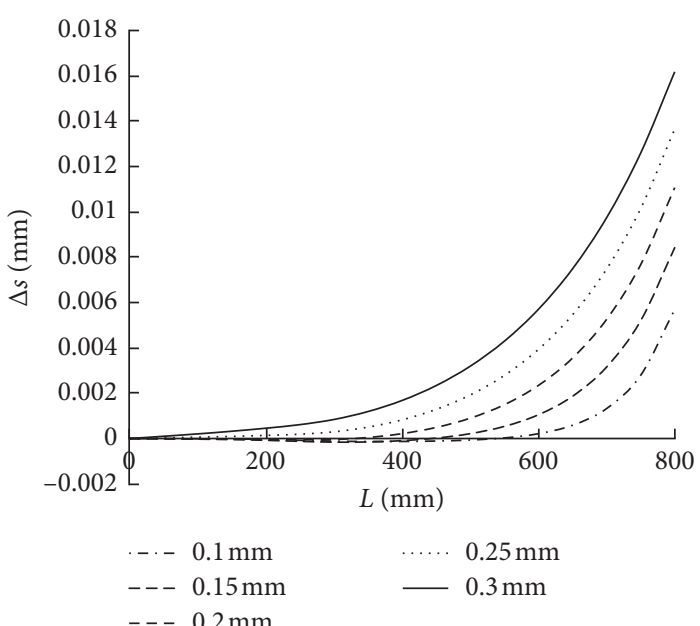

(b)

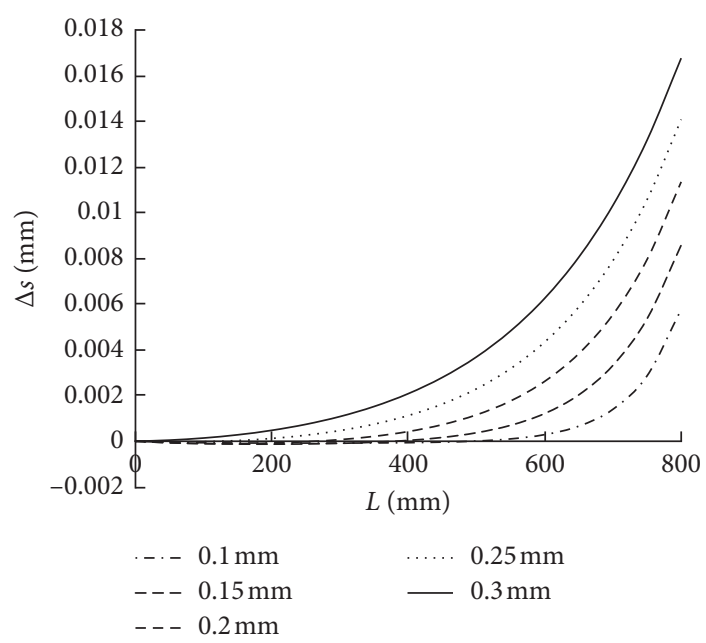

(c)

Figure 9: The effect of CFRP sheet's thickness on the interface slip. (a) Uniform load. (b) Symmetrical concentrated load. (c) Midspan concentrated load. 


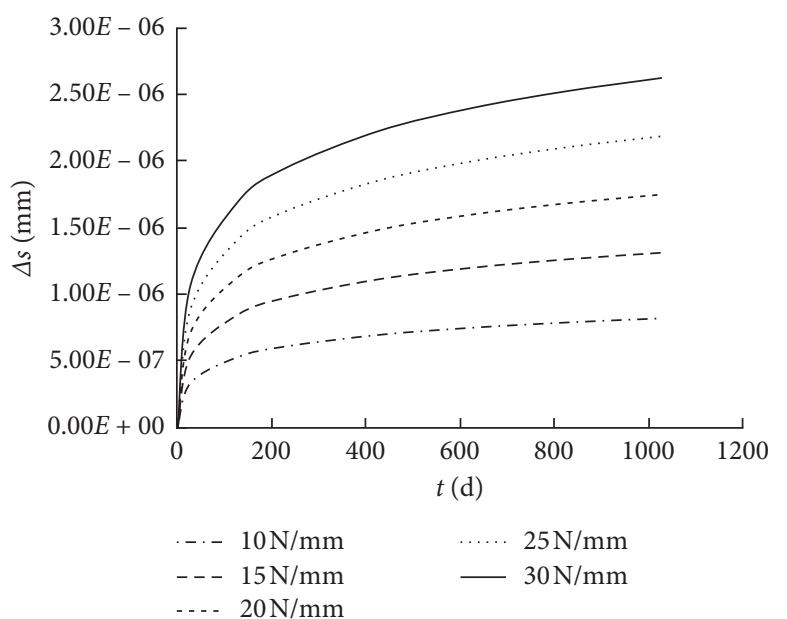

(a)

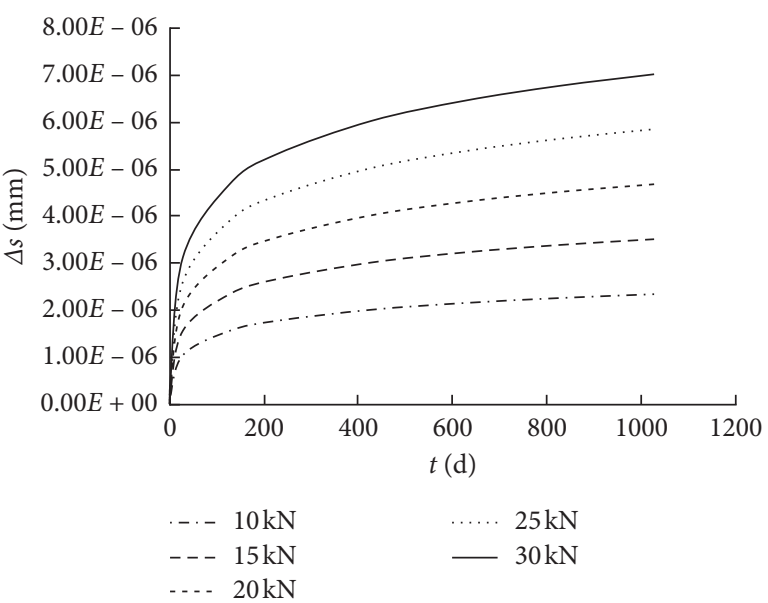

(b)

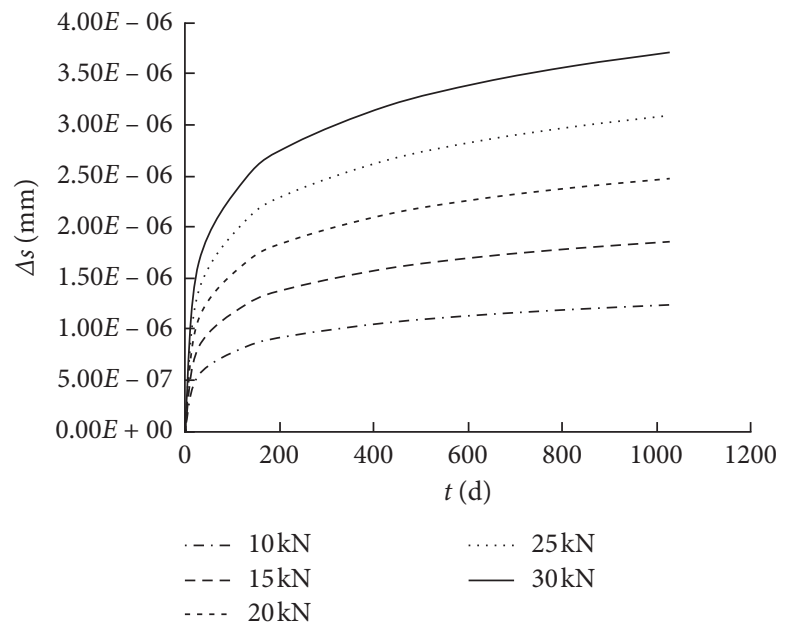

(c)

Figure 10: The effect of loading under creep on the interface slip. (a) Uniform load. (b) Symmetrical concentrated load. (c) Midspan concentrated load.

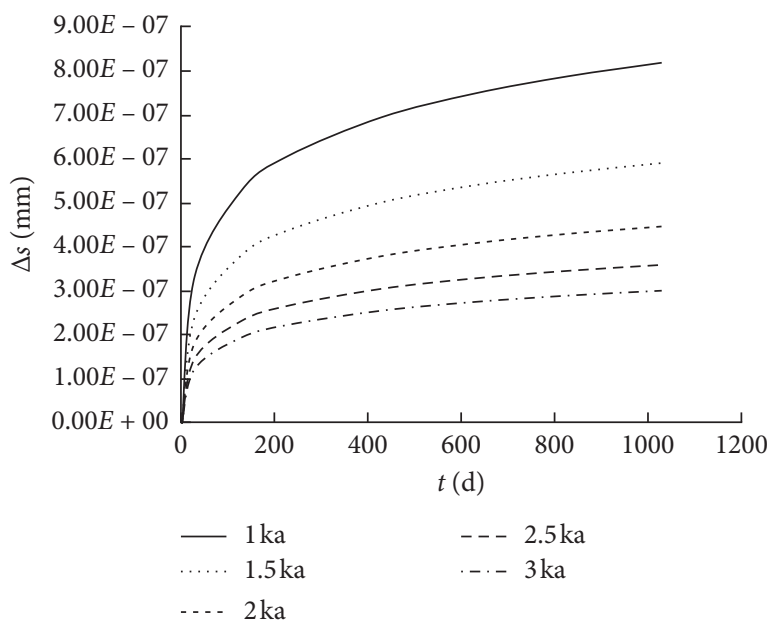

(a)

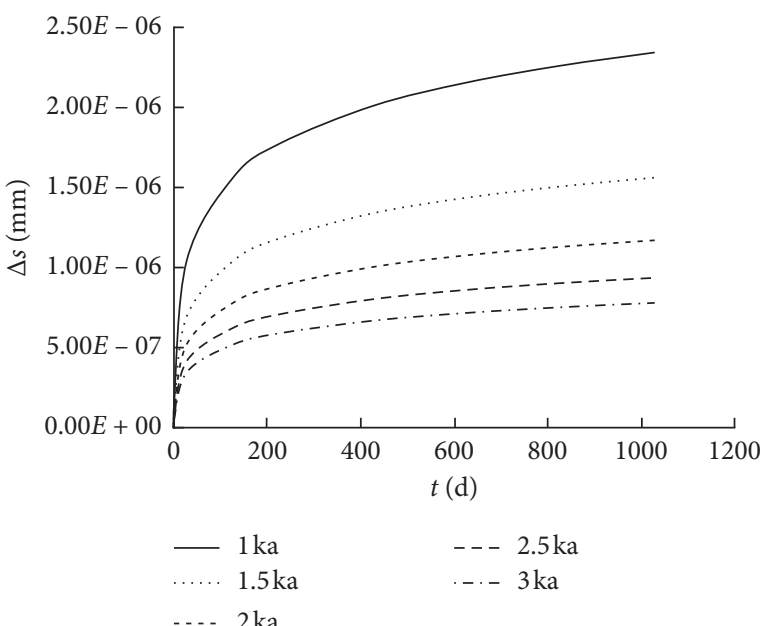

(b)

Figure 11: Continued. 


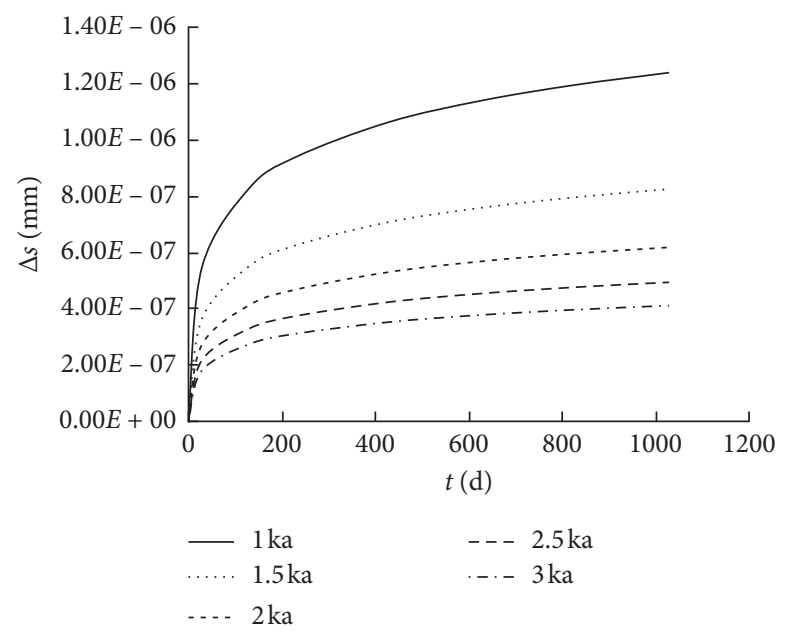

(c)

Figure 11: The effect of adhesive stiffness under creep on the interface slip. (a) Uniform load. (b) Symmetrical concentrated load. (c) Midspan concentrated load.

However, the change of the interface slip decreased with the increase of stiffness. When the stiffness reached about $10 \mathrm{ka}$, the variation of the stiffness on the slip was basically unchanged.

\section{Conclusion}

(1) The formulas of the interface slip of the steel beam reinforced with the CFRP sheet under the action of temperature and creep were given by using the elastic method and energy method, respectively. By comparing the results between the finite element calculation and the formula calculation, it showed that the two results were in good agreement and indicated that the formula was correct and can be used to calculate the interface slip between the steel beam and the CFRP sheet.

(2) The results of formulas showed that when the creep was not taken into account, the interface slip was the maximum at the end of the beam and the minimum at the middle of the span. When the stiffness reached about $3 \mathrm{ka}$, the slip was basically unchanged. When the temperature increased to $5^{\circ} \mathrm{C}$, the slip increased about $0.005 \mathrm{~mm}$. And when the thickness of the CFRP sheet increased to $0.05 \mathrm{~mm}$, the slip increased about $0.003 \mathrm{~mm}$.

(3) The results of formulas also showed that when the creep was taken into account, the interface slip increased with the increase of load and decreased with the increase of stiffness. When the load increased to $5 \mathrm{~N} / \mathrm{mm}$ or $5 \mathrm{kN}$, the slip increased about $4.4 \times 10^{-7} \mathrm{~mm}, \quad 1.2 \times 10^{-6} \mathrm{~mm}$, and $6.2 \times 10^{-7} \mathrm{~mm}$, respectively. The change of the interface slip decreased with the increase of stiffness. When the stiffness reached about $10 \mathrm{ka}$, the variation of the stiffness on the slip was basically unchanged.

\section{Data Availability}

Some or all data, models, or codes generated during this study are available from the corresponding author upon request.

\section{Conflicts of Interest}

The authors declare that they have no conflicts of interest.

\section{References}

[1] M. Yao, Effect of Temperature and Loading Rate on Bond Characteristics between FRP Sheet and Steel Plate, Hunan University, Changsha, China, 2016.

[2] T.-C. Nguyen, Y. Bai, X.-L. Zhao, and R. Al-Mahaidi, "Durability of steel/CFRP double strap joints exposed to sea water, cyclic temperature and humidity," Composite Structures, vol. 94, no. 5, pp. 1834-1845, 2012.

[3] T. Yu, D. Fernando, J. G. Teng, and X. L. Zhao, "Experimental study on CFRP-to-steel bonded interfaces," Composites Part B: Engineering, vol. 43, no. 5, pp. 2279-2289, 2012.

[4] X. Jiang, The Theoretical Analysis and Experimental Study on Interfacial Properties of Steel Beams Reinforced by CFRP Plates, Hefei University of Technology, Hefei, China, 2013.

[5] D. Fernando, T. Yu, and J. G. Teng, "Behavior of CFRP laminates bonded to a steel substrate using a ductile adhesive," American Society of Civil Engineers, vol. 18, no. 2, pp. 411-419, 2013.

[6] A. Al-Shawaf and X.-L. Zhao, "Adhesive rheology impact on wet lay-up CFRP/steel joints' behaviour under infrastructural subzero exposures," Composites Part B: Engineering, vol. 47, pp. 207-219, 2013.

[7] X.-L. Zhao, Y. Bai, and R. Al-Mahaidi, "Effect of dynamic loading and environmental conditions on the bond between CFRP and steel," Journal of Composites for Construction, vol. 18, no. 3, Article ID A4013005, 2014.

[8] Y. Xue, Experimental Research on Bond Performance of CFRPSteel Interface, Southeast University, Nanjing, China, 2014. 
[9] J. Teng, D. Fernando, and T. Yu, "Finite element modeling of debonding failures in steel beams flexurally strengthened with CFRP laminates," Engineering Structures, vol. 86, pp. 213-224, 2015.

[10] J. Yuan, Experimental Research and Numerical Simulations on H-Shaped Steel Beam Strengthened with CFRP, Shanghai Jiao Tong University, Shanghai, China, 2011.

[11] H.-T. Wang, G. Wu, Y.-T. Dai, and X.-Y. He, "Experimental Study on bond behavior between CFRP plates and steel substrates using digital image correlation," Journal of Composites for Construction, vol. 20, no. 6, Article ID 04016054, 2016.

[12] M. Heshmati, R. Haghani, and M. Al-Emrani, "Durability of bonded FRP-to-steel joints: effects of moisture, de-icing salt solution, temperature and FRP type," Composites Part B: Engineering, vol. 119, pp. 153-167, 2017.

[13] C. Li, L. Ke, Z. Chen, J. He, and N. Luo, "Experimental study and numerical simulation for bond behavior of interface between CFRP and steel," Acta Materiae Compositae Sinica, vol. 35, no. 12, pp. 3534-3546, 2018.

[14] Y. Pang, Study on the Bond Behavior of CFRP-Steel Interfaces Under Extreme Service Environment, Southeast University, Nanjing, China, 2019.

[15] F. Wei, D. Zhu, H. Wang, and Z. Bian, "Experimental study on bond behavior of CFRP plate-steel interface in freeze-thaw environment," Journal of Southeast University, vol. 50, no. 5, pp. 803-807, 2020.

[16] B. Xu, Y. Yao, Y. Qian, M. Ma, and S. Song, "Experiment on bonding behavior of CFRP-steel interface with load history," Journal of Harbin Institute of Technology, vol. 52, no. 9, pp. 167-175, 2020.

[17] B. Xu, Y. Yao, Y. Qian, C. Zhang, and S. Song, "Creep constitutive model of CFRP-steel interface under shear stress," Journal of Harbin Institute of Technology, vol. 53, no. 3, pp. 170-177, 2021.

[18] S. Liu, Study on Mechanical Properties of Corroded Steel Beam Strengthened with CFRP Sheet, Northeastern University, Shenyang, China, 2010.

[19] JTG 3662-2018, Specifications for Design of Highway Reinforced Concrete and Prestressed Concrete Bridge and Culverts, People's Communications Press, Beijing, China, 2018.

[20] M. Nakada and Y. Miyano, "Statistical creep failure time of unidirectional CFRP," Experimental Mechanics, vol. 56, no. 4, pp. 653-658, 2016.

[21] L. Wang, Prestressed Composite Structure of Steel and Concrete, Science Press, Beijing, China, 2008. 